\title{
Visible-infrared self-amplified spontaneous emission amplifier free electron laser undulator
}

\author{
Roger Carr, Max Cornacchia, Paul Emma, Heinz-Dieter Nuhn, Ben Poling, and Robert Ruland \\ Stanford Linear Accelerator Center, 2575 Sand Hill Road, Menlo Park, California 94025 \\ Erik Johnson, George Rakowsky, and John Skaritka \\ Brookhaven National Laboratory, Upton, New York 11973 \\ Steve Lidia \\ Lawrence Berkeley National Laboratory, Berkeley, California 94720 \\ Pat Duffy and Marcus Libkind \\ Lawrence Livermore National Laboratory, Livermore, California 94550
}

\author{
Pedro Frigola, Alex Murokh, Claudio Pellegrini, James Rosenzweig, and Aaron Tremaine \\ University of California, Los Angeles, California 90024 \\ (Received 13 April 2001; published 13 December 2001)
}

\begin{abstract}
The visible-infrared self-amplified spontaneous emission amplifier (VISA) free electron laser (FEL) is an experimental device designed to show self-amplified spontaneous emission (SASE) to saturation in the near infrared to visible light energy range. It generates a resonant wavelength output from 800-600 nm, so that silicon detectors may be used to characterize the optical properties of the FEL radiation. VISA is designed to show how SASE FEL theory corresponds with experiment in this wavelength range, using an electron beam with emittance close to that planned for the future Linear Coherent Light Source at SLAC. VISA comprises a $4 \mathrm{~m}$ pure permanent magnet undulator with four $99 \mathrm{~cm}$ segments, each of 55 periods, $18 \mathrm{~mm}$ long. The undulator has distributed focusing built into it, to reduce the average beta function of the $70-85 \mathrm{MeV}$ electron beam to about $30 \mathrm{~cm}$. There are four FODO cells per segment. The permanent magnet focusing lattice consists of blocks mounted on either side of the electron beam, in the undulator gap. The most important undulator error parameter for a free electron laser is the trajectory walk-off, or lack of overlap of the photon and electron beams. Using pulsed wire magnet measurements and magnet shimming, we were able to control trajectory walk-off to less than $\pm 50 \mu \mathrm{m}$ per field gain length.
\end{abstract}

DOI: $10.1103 /$ PhysRevSTAB.4.122402

PACS numbers: 41.60.Cr, 41.60.Ap

\section{INTRODUCTION}

This work arose from a long-range goal to design and build an x-ray free electron laser (FEL) based on a linac electron source and a single pass undulator that will generate FEL radiation starting from noise. The eventual goal is the Linear Coherent Light Source (LCLS), a $1.5 \AA$ selfamplified spontaneous emission (SASE) FEL based on a $15 \mathrm{GeV}$ linac at SLAC [1]. The single pass design is required for a mirrorless $\mathrm{x}$ ray, and it must amplify noise because we have no coherent seed radiation at x-ray wavelengths. Therefore, it is essential to understand the physics of self-amplified spontaneous emission in these devices [2]. SASE gain has been demonstrated from noise start-up at a $15 \mu \mathrm{m}$ wavelength, but the $2 \mathrm{~m}, 100$ period undulator we used was not long enough to reach saturation [3]. Also, at that wavelength, detectors are not easily available to investigate the detailed structure of the gain mechanism. A preferable wavelength range would be in the visible or near infrared, where silicon detectors can be used and where Fourier transform methods are available to analyze the time structure of the FEL radiation. It was for this reason that we built the visible-infrared SASE amplifier (VISA) FEL. It is installed in the Accelerator Test Facility (ATF) linac at Brookhaven National Laboratory, which has been upgraded to an energy range of $70-85 \mathrm{MeV}$. The ATF has a laser photocathode gun capable of delivering $1.5 \mathrm{psec}, 200$ A electron pulses with a normalized emittance of $2 \pi \mathrm{mm} \mathrm{mrad}$. The first experimental tests were completed recently [4].

There are several other groups experimenting with SASE FELs. The LEUTL experiment of the Advanced Photon Source at Argonne National Laboratory has achieved SASE lasing at 530 and $385 \mathrm{~nm}$ using nine $2.4 \mathrm{~m}$ long, $33 \mathrm{~mm}$ period, hybrid undulators originally built for storage ring use, with focusing magnets between the undulators [5]. LEUTL used a $217 \mathrm{MeV}$ electron beam with $8.5 \pi \mathrm{mm}$ mrad normalized emittance for the $530 \mathrm{~nm}$ experiment. The TESLA test facility at DESY has also achieved SASE lasing at $109 \mathrm{~nm}$, with a gain of about 3000 . They used three $4.5 \mathrm{~m}$ undulators of $27.3 \mathrm{~mm}$ period length with $12 \mathrm{~T} / \mathrm{m}$ gradient distributed focusing [6]. They used a $233 \mathrm{MeV}$ electron beam with $6 \pm 3 \pi \mathrm{mm} \mathrm{mrad}$ normalized emittance. The VISA project was designed 
to gain experience with the LCLS parameters such as electron beam emittance, beam pipe diameter, etc., which are smaller than the other designs. A comparison of the three designs is presented in [7].

\section{SPECIFICATIONS}

Based on GINGER, FRED3D, and semiempirical model simulations, the FEL physics requires a certain set of parameters and tolerances on those parameters, as shown in Table I [8]. Values separated by a slash (/) represent values at 72.6 and $83.84 \mathrm{MeV}$ or $800 / 600 \mathrm{~nm}$ wavelengths.

\section{UNDULATOR STRUCTURE}

The VISA undulator has a resonant wavelength of $800 \mathrm{~nm}(600 \mathrm{~nm})$ at $72.6 \mathrm{MeV}(83.8 \mathrm{MeV})$. The corresponding saturation length is calculated numerically to be $3.4 \mathrm{~m}(3.8 \mathrm{~m})$, for an ideal undulator with $18 \mathrm{~mm}$ period and a maximum magnetic field of $0.75 \mathrm{~T}$. Therefore, we decided to build an undulator $4 \mathrm{~m}$ long. The period length and field strength are optimized from numerical calculations of FEL performance. These parameters can be achieved with a pure Halbach permanent magnet approach with no permeable materials, $\mathrm{NdFeB}$ magnets with $\mathrm{Br}=1.25 \mathrm{~T}$, and a fixed gap of $6 \mathrm{~mm} \mathrm{[9].} \mathrm{The}$
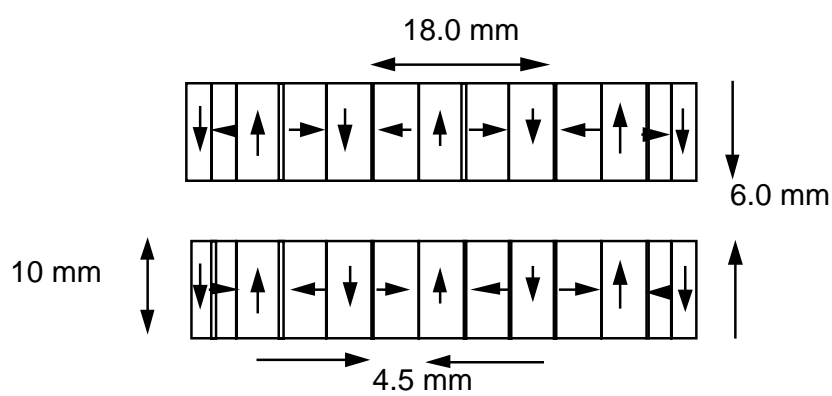

FIG. 1. Schematic side view of two periods of the VISA undulator structure, showing a symmetric two-half-block termination scheme. Arrows within the magnet blocks indicate the direction of magnetization.

magnet blocks are $10 \mathrm{~mm}$ high and $4.5 \mathrm{~mm}$ thick; the rectangular shape gives $23 \%$ more field than square cross section blocks would yield. Figure 1 shows the magnetic scheme for the VISA undulator.

The symmetric two-half-block termination scheme results in negligible trajectory offset, as shown in Fig. 2

The $4 \mathrm{~m}$ VISA undulator is built from the beginning as part of a subsequent experiment at the Source Development Laboratory at Brookhaven National Laboratory that calls for a $6 \mathrm{~m}$ undulator with the same period length and gap. Therefore, we modularized the magnetic structure

TABLE I. The VISA undulator specifications.

\begin{tabular}{|c|c|c|}
\hline Parameter & Symbol or formula & Specification \\
\hline Period length & $\lambda u$ & $18 \mathrm{~mm}$ \\
\hline Number of periods & $N$ & $220(55 /$ segment $)$ \\
\hline Magnetic gap & $g$ & $6.0 \mathrm{~mm}$ \\
\hline Maximum $B$ field & $B_{\max }$ & $0.75 \mathrm{~T}$ \\
\hline$B$ field error & $\Delta B / B_{\max }$ & $0.4 \%$ \\
\hline Undulator parameter & $K(\mathrm{aw})$ & $1.26 / 0.89$ \\
\hline Peak-to-peak wiggle amplitude & $a_{0}=K /(\gamma k)$ & $51 / 44 \mu \mathrm{m}$ \\
\hline Length of $F$ and $D$ & $L_{F}=L_{D}$ & $90 \mathrm{~mm}$ effective \\
\hline Length of FODO cell & $L_{\text {FODO }}$ & $247.5 \mathrm{~mm}$ \\
\hline Phase advance/cell & $\Phi_{\text {cell }}$ & $61^{\circ} / 52^{\circ}$ \\
\hline Phase error & $\delta \phi$ & $2^{\circ}$ \\
\hline Focusing gradient & $g_{f}$ & $33.3 \mathrm{~T} / \mathrm{m}$ \\
\hline Focusing gradient error & $\delta_{g_{f} / g_{f}}$ & $0.2 \%$ \\
\hline Maximum beta function & $\beta_{\max }$ & $408 / 431 \mathrm{~m}$ \\
\hline Minimum beta function & $\beta_{\min }$ & $134 / 168 \mathrm{~m}$ \\
\hline Beta function modulation & $\beta_{\max }-\beta_{\min } / \beta_{\mathrm{avg}}$ & $101 \% / 88 \%$ \\
\hline Segment straightness & & $\pm 50 \mu \mathrm{m} / \mathrm{m}$ \\
\hline Horizontal segment location & & $\pm 500 \mu \mathrm{m}$ \\
\hline Vertical segment location & & $\pm 100 \mu \mathrm{m}$ \\
\hline Axial segment location & & $\pm 500 \mu \mathrm{m}$ \\
\hline Segment roll & & $\pm 100 \mu \mathrm{rad}$ \\
\hline Segment pitch & & $\pm 100 \mu \mathrm{rad}$ \\
\hline Segment yaw & & $\pm 50 \mu \mathrm{rad}$ \\
\hline Trim coil field & & $\pm 50 \mathrm{G}$ \\
\hline Effective trim coil length & & $200 \mathrm{~mm}$ \\
\hline Maximum trim kick & & $4.1 / 3.6 \mu \mathrm{rad}$ \\
\hline Required vacuum pressure & & $10^{-6}$ Torr \\
\hline Vacuum pump capacity & & $4500 \mathrm{l} / \mathrm{sec}$ \\
\hline
\end{tabular}




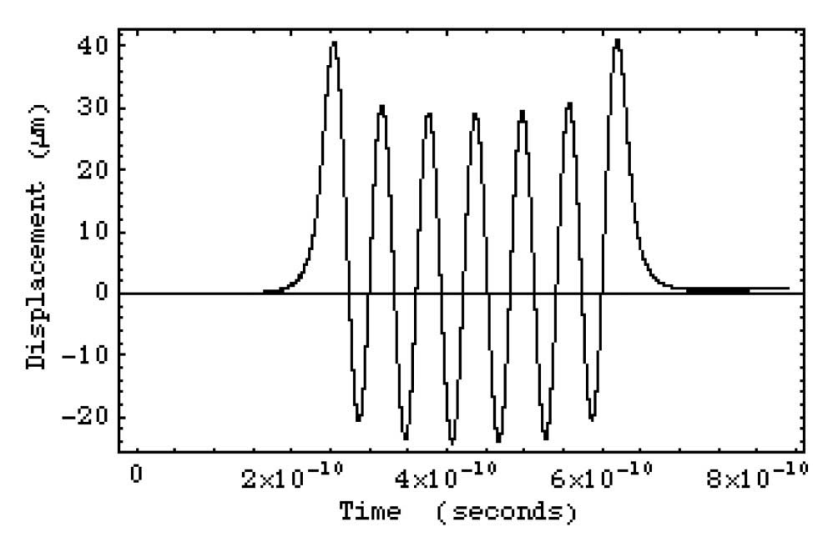

FIG. 2. Numerical trajectory calculation for six periods of VISA undulator, with symmetric two-half-block termination. The wiggle amplitude, peak to peak, is about half the beam diameter. into $99 \mathrm{~cm}$ segments. Four of these 55 period segments comprise VISA, and they are butted together. If onewavelength drift spaces, $\lambda_{u}\left(1+K^{2} / 2\right)=32.3 \mathrm{~mm}$ long, were allowed between segments, the short Rayleigh length $(30-38 \mathrm{~mm})$ of this FEL would allow unacceptable diffraction losses. There may be gaps of a fraction of a millimeter between segments, which cause a trajectory phase error, but this should not seriously harm the FEL gain. Figure 3 shows the entire $4 \mathrm{~m}$ undulator.

The electron beam in VISA has an rms radius of $60 \mathrm{~mm}$, and numerical simulations show that saturation length is adversely affected if the trajectory walks off a straight line by more than $\pm 50 \mu \mathrm{m}$ per field gain length of $34 \mathrm{~cm}$ $(38 \mathrm{~cm})$ at $72 \mathrm{MeV}(83 \mathrm{MeV})$. A magnetic field with rms errors of less than $0.4 \%$ is required in order to achieve this trajectory walk-off tolerance.

Natural focusing is too weak for a $4 \mathrm{~m}$ undulator to saturate at these wavelengths, and we are constrained by the ATF laboratory layout to this length. Therefore we had to add strong focusing to the undulator. To achieve an

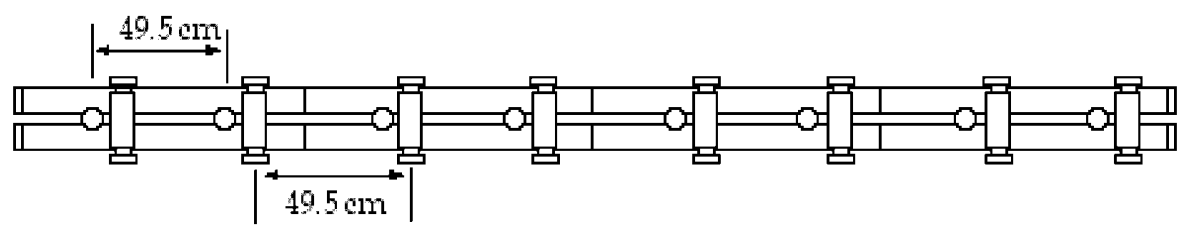

FIG. 3. Schematic side view of the VISA undulator, with end terminating magnets on both ends, pop-in diagnostic ports (circles), and steering trim coils (vertical oblong shapes).

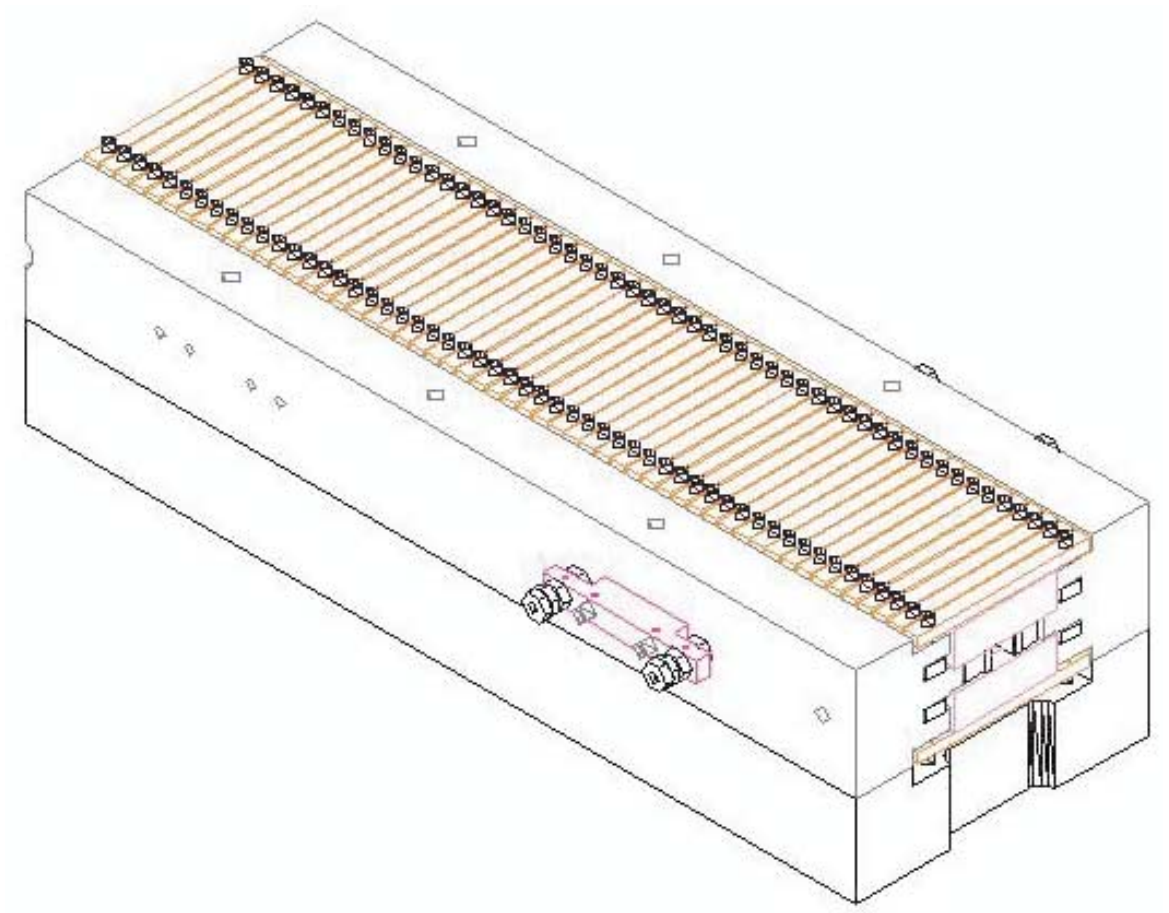

FIG. 4. (Color) Schematic view of the VISA undulator, showing dipole magnets (violet) with individual hold down bars (orange). The spacers assure a precise gap between the dipole magnets. The fixture on the side is the adjustor mechanism for the quadrupole magnet assemblies, whose ends show in the gap between dipole magnets. 


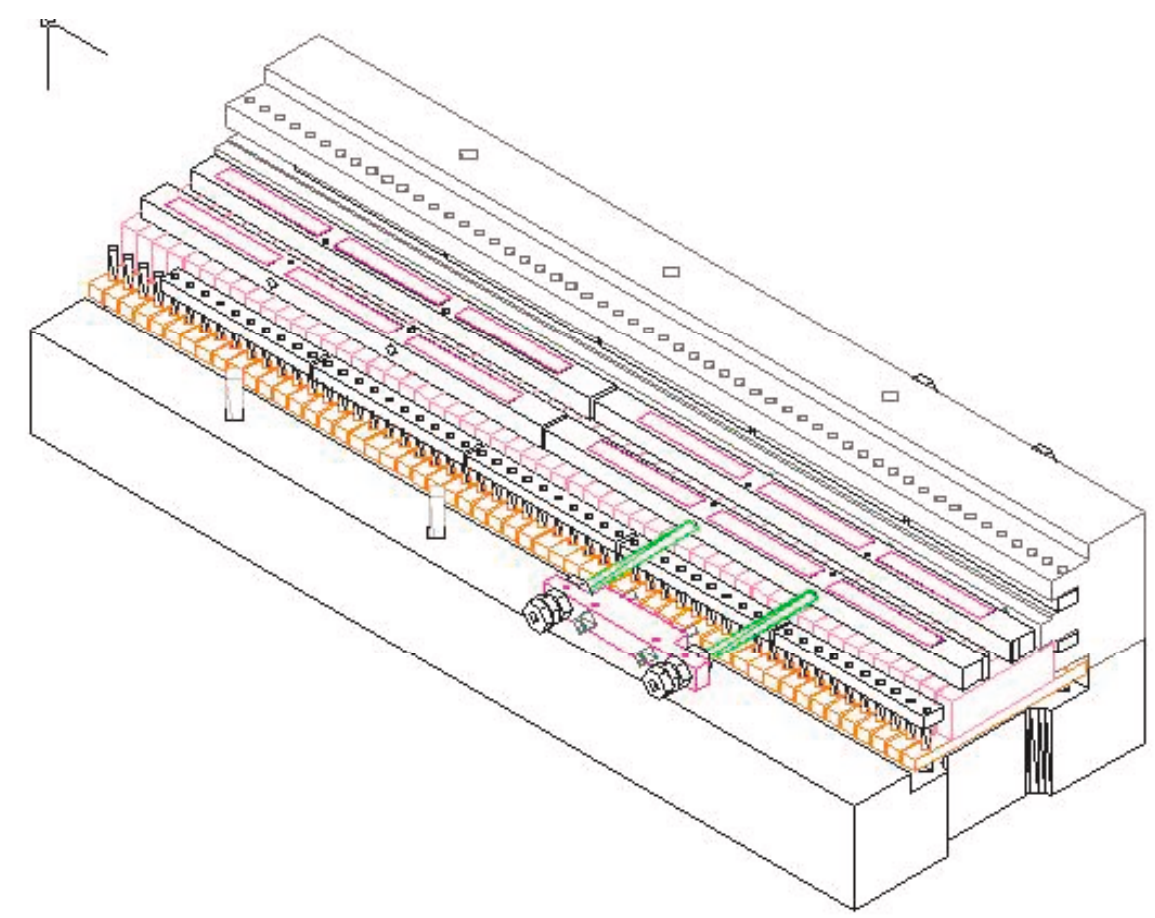

FIG. 5. (Color) Cutaway view of the VISA undulator showing the lower row of dipole magnets and the quadrupole assemblies above them. The adjustor assembly with green rods was first designed only to provide horizontal motion and was later modified with cams on the ends of the rods to move the focusing magnet assemblies in the vertical.

average beta function of $27-30 \mathrm{~cm}$ between 72 and $83 \mathrm{MeV}$, distributed focusing is preferred to lumped focusing, so we decided to put a FODO lattice with four cells per segment into the undulator. Figure 4 shows the overall mechanical structure of the undulator.

The focusing magnet's blocks are $30 \mathrm{~mm}$ long $\times 4 \mathrm{~mm}$ wide horizontally $\times 4.5 \mathrm{~mm}$ high, arranged in assemblies of three blocks on each side of the beam axis. There are four FODO cells per undulator segment, and thus 16 magnet assemblies, two for each $\mathrm{F}$ or $\mathrm{D}$. The assemblies are $99 \mathrm{~mm}$ long (because there are spaces between blocks), and there is a horizontal gap of $10.25 \mathrm{~mm}$ between them. The permanent magnet material $(\mathrm{NdFeB})$ has a remanence $\mathrm{Br}=1.25 \mathrm{~T}$, so they generate gradients of $33 \mathrm{~T} / \mathrm{m}$ on axis. The material has $\mathrm{Hci}>20 \mathrm{kOe}$, so that it resists demagnetization in this geometry.

The focusing assemblies comprise magnet blocks sandwiched between aluminum bars, so that the horizontal aperture for the beam is about $7 \mathrm{~mm}$. The surfaces of the undulator magnets above and below the aperture are covered by a $25 \mu \mathrm{m}$ Ni foil that provides a smooth, high conductivity surface to reduce resistive wall wakefields and wakefield effects caused by the narrow transverse gaps between adjacent magnets.

In the original design, the focusing magnet assemblies were attached to the keepers by means of horizontal rods that were screwed into the assemblies and had their ends held outside the keepers by nuts on two sides of a bracket. They were placed into the segment before the dipole mag- nets, aligned against a precision ground spacer bar, and fixed in place. The assemblies were made $0.1 \mathrm{~mm}$ thinner than the $6 \mathrm{~mm}$ gap, so that they were not held tightly when the dipoles were installed. Figure 5 shows the details of the focusing magnet system.

\section{MAGNETIC MEASUREMENTS AND ERROR MINIMIZATION}

The magnetic field errors of a pure permanent magnet undulator can be controlled in several ways. First, magnet material is chosen within a certain tolerance band on the magnetic moments and the direction of magnetization of each block. We specified $\mathrm{NdFeB}$ material with $\mathrm{Br}=1.25 \mathrm{~T}$, moment errors of no more than $1.5 \%$ of $\mathrm{Br}$ in each of the three principal axes of the rectangular blocks, and direction error of no more than $1.5^{\circ}$. After Helmholtz coil measurements are made of the three principal moments of all blocks, a sorting algorithm is executed on the data.

We employ a technique called threshold acceptance, which is similar to simulated annealing [10]. Briefly, a random arrangement of magnets is improved by trading magnets pairwise. If a trade either improves a merit function or degrades it by no more than a threshold value the trade is kept. The threshold is gradually lowered, and finally a local minimum is found by letting the threshold be zero. The merit function we use is a simple vector addition of magnetic field errors with nearest neighbor 
magnets. Several sorts are done starting from different random states and the best one is chosen. We can reduce starting error values of $1.5 \%$ down to about $0.4 \%$, which meets our requirements.

After sorting and assembly, the undulator assemblies have magnetic errors from magnetic measurements and mechanical imperfections, so we employ magnetic shimming to improve the trajectory in each segment. This work was performed by the magnetic measurements group of the NSLS at BNL, using the pulsed wire technique, as shown in Fig. 6. This technique is well established for simple undulators [11], but it is more complicated in our case where quadrupole focusing fields are superposed over the undulator dipole fields. The full description of the magnetic measurements is given in Ref. [12], so we give only a condensed version here.

First, we find the axis of the quadrupole focusing field. A short rise time current pulse is put into the wire (to observe the first integral of the field), and the resulting mechanical vibrations are detected by an optical pickup. We translate the wire transversely to seek a null in the 24.75 $\mathrm{cm}$ period signal from the FODO lattice. To enhance this signal, we suppress the larger $1.8 \mathrm{~cm}$ signal from the dipole magnet fields by low pass filtering or by Fourier transform techniques. From tests on a prototype VISA magnet, we demonstrated that we can locate the quadrupole axis to $\pm 20 \mu \mathrm{m}$. The undulator sections are designed to be rotated $90^{\circ}$, so that separate $x$ and $y$ measurements can be made in the horizontal plane, to eliminate the effect of wire sag.

Having found the quadrupole axis, we measure its position mechanically with a precise wire finder that is referenced to tooling balls attached to each end of the undulator segment. Micrometers mounted on the finder are moved so that they make electrical contact with the wire. We find this is repeatable to about $\pm 5 \mu \mathrm{m}$. The positions of the tooling balls relative to each other were found on a coordinate measuring machine. Also, the wire finder was calibrated to determine the micrometer readings of the nominal centerline. The best axis wire positions and the calibration offsets were then used to align the undulators when they were installed in the vacuum vessel.

The next step was to observe the second integral of the field (i.e., the trajectory), using a current step (in practice, a long, square pulse). We then added shim magnets outboard from the dipole array to correct errors in the trajectory. The shim magnets are small $3 \mathrm{~mm} \times 3 \mathrm{~mm} \times 2 \mathrm{~mm}$ blocks of $\mathrm{NdFeB}$ that are used in fours, as shown in Fig. 7. The blocks are mounted in pairs on movers that extend into wells machined through the strongbacks shown in Fig. 4.

The BNL pulsed wire system is capable of measuring a $2 \mathrm{~m}$ undulator. After shimming individual $1 \mathrm{~m}$ segments, we set up pairs of segments, butted together, and shim the trajectory (as measured by the pulsed wire) across the joint, to minimize trajectory errors. A typical set of the data is shown in Fig. 8.

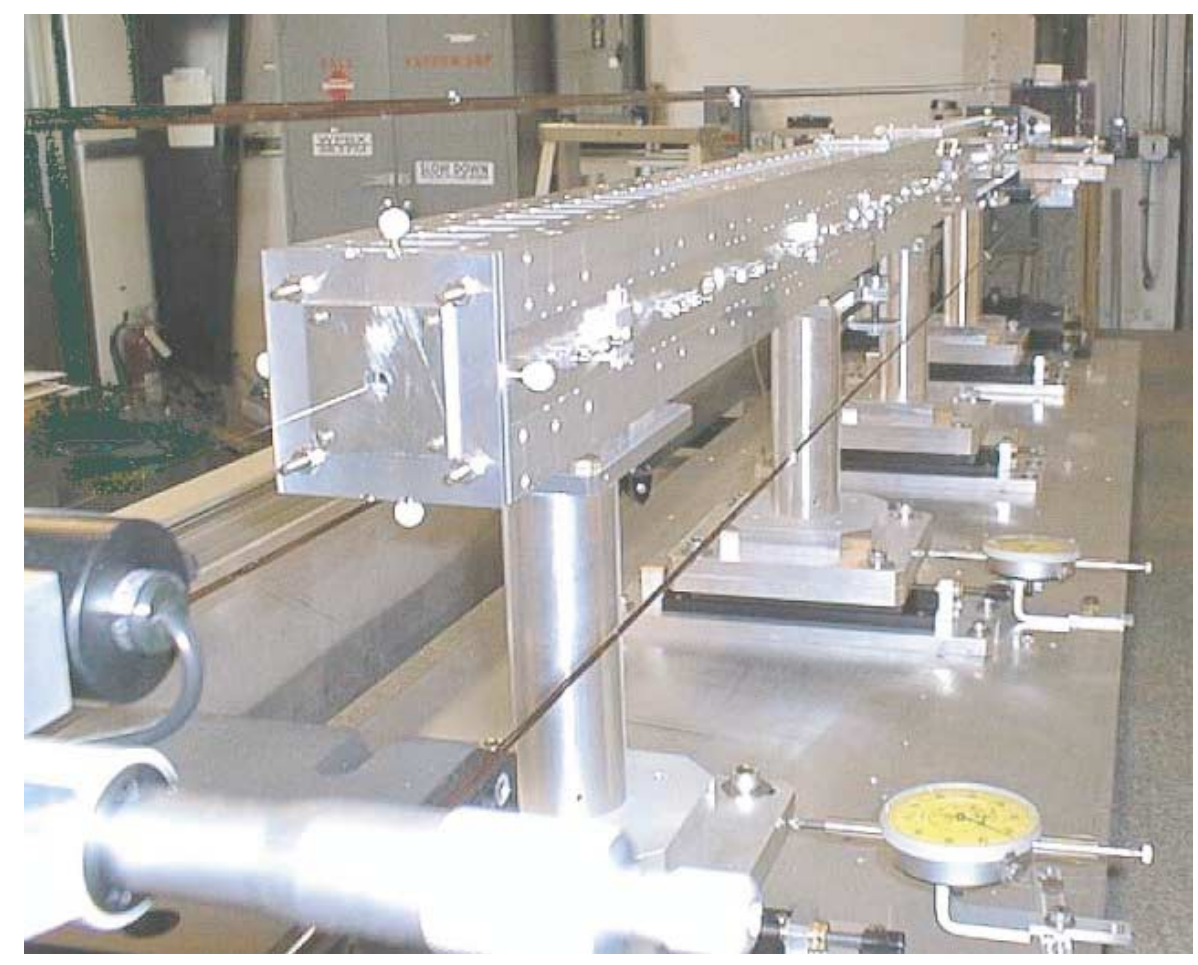

FIG. 6. (Color) Two segments of the VISA undulator mounted on the stretched wire testing stand. The wire may be seen passing down the axis of the undulator. 

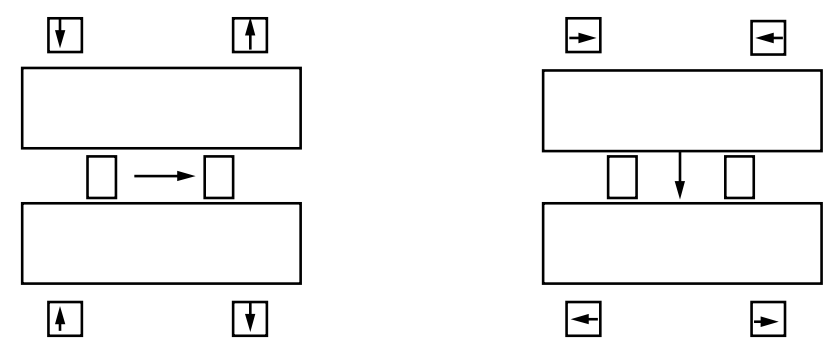

FIG. 7. Schematic end view of the VISA undulator, showing placement of shim magnets in groups of four. With magnetizations arranged as at left, the shim magnets create a net horizontal field on axis, and on the right they create vertical field. By varying the vertical positions of these magnets, we obtain a tuning range of about $3-35 \mathrm{Gcm}$.

By adjusting the horizontal positions of the focusing magnets, the horizontal trajectory could be made to walkoff by less than $10 \%$ of the wiggle amplitude (see Fig. 8) over the length of a segment, which is far better than is required for the FEL. We concluded that most of the trajectory error was due to the positioning of the focusing magnets, since they have a relatively strong gradient. Though we employed some shim magnets in addition, most of the corrections were achieved using only the focusing magnets.

This still left a need to compensate for the vertical trajectory errors, which were approximately as large as the horizontal errors. Shim magnets that generated a horizontal field were found too weak to correct all vertical errors, so we attached larger shims directly to the sides of the segments to provide horizontal field corrections. With these magnets, we corrected the vertical errors about as well as the horizontal errors.

In hindsight, we found that trajectory corrections were much more readily achieved using focusing magnets than shim magnets. We reasoned that we could correct both vertical and horizontal errors by moving the focusing magnets in the vertical and the horizontal, so as to obviate the need for shim magnets. Because of its value for future

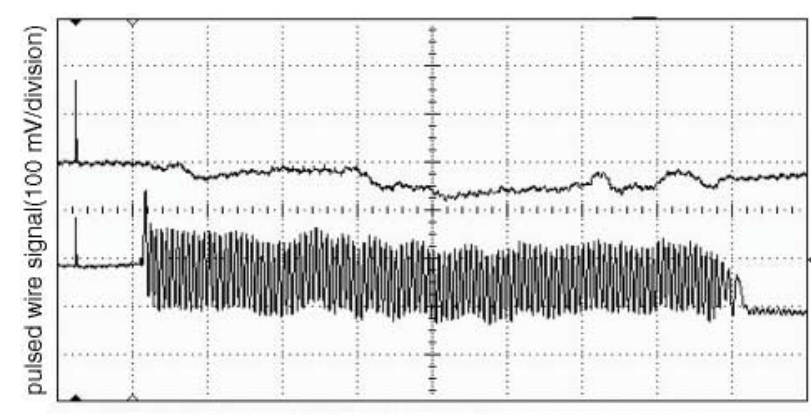

Distance along axis of undulator $-25 \mathrm{~cm} /$ division

FIG. 8. The horizontal (lower curve) and vertical (upper curve) trajectories, as measured by the stretched wire apparatus. The graph shows data for two undulator sections butted together, after shimming has been done to compensate for errors at the joint between them. The overall error is well within $10 \%$ of the wiggle amplitude per gain length.
FEL designs, we tested this concept using a spare undulator segment that was not incorporated into VISA. The rods holding the focusing magnets were machined to have a cam motion, with their ends eccentric by $0.33 \mathrm{~mm}$. Focusing magnet keepers were made out of single blocks of 7075 aluminum, and the magnets were held in place by small dots of Torr-Seal ${ }^{\circledR}$ adhesive. The focusing magnets were simply pressed against a thin floor machined in the holder. The holder was only $5.15 \mathrm{~mm}$ high, leaving a gap of $0.42 \mathrm{~mm}$ above and below when centered. The gap has some disadvantages in terms of the rf environment, but it allows us to center the trajectory very well. In preliminary tests, we found that we could perform all corrections using only adjustments of the focusing magnets, with no shims.

\section{VACUUM VESSEL}

There were two possible approaches to the vacuum vessel design, an external box or an internal beam pipe. We chose the external box, partly because it is the approach we used in the Los Alamos experiment, and partly because the fabrication of a $6 \mathrm{~mm}$ "soda straw" beam pipe, with ports for diagnostics, was an unknown. In retrospect, we found that the external vessel made alignment of the undulator very difficult. It also made difficult the mounting of the diagnostics because they were attached to the vessel and not to the undulator. It was necessary to decouple the external vessel from the undulator mechanically, so that no stresses induced by pumpdown would alter the position of the undulator, which was aligned by means of measurements taken before pumpdown.

The vacuum chamber was supported on a five-legged table with a $25 \mathrm{~mm}$ thick ground aluminum top. The undulator was supported by eight transverse slides that sat atop this table, on which were mounted posts that held the magnet segments near each end. A flexible welded bellows was used to provide a vacuum seal between the slide and the vessel, and, it was hoped, to decouple the two mechanically. As it turned out, the upward force of the vacuum system on the slides caused them to move slightly during pumpdown, destroying the alignment of the undulator. The slides were replaced by a parallelogram flexure mover operated by push-push screws, and which 


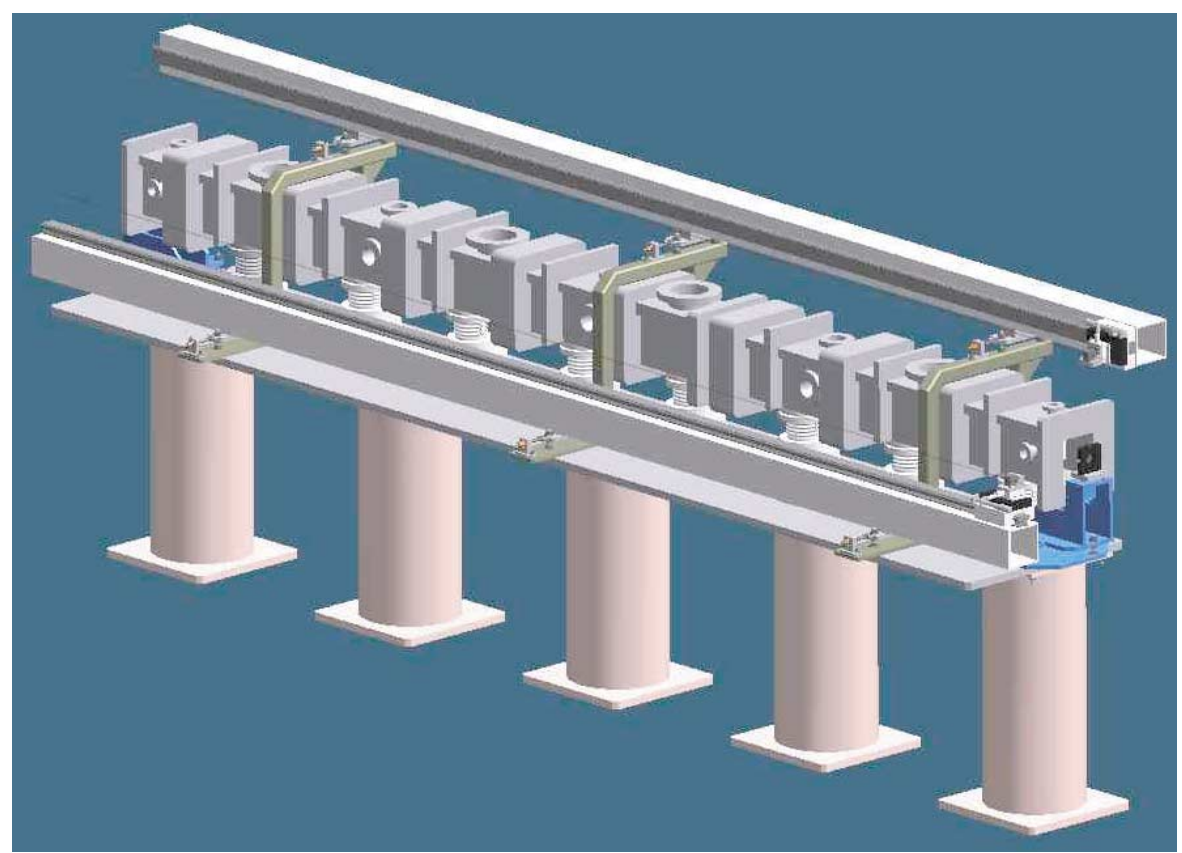

FIG. 9. (Color) Computer-aided design drawing of the VISA undulator vacuum vessel, support table, and rails for the straightness interferometer measurements. The thick square objects around the vessel represent iron-core window-frame steering correction magnets. The thin square objects represent stiffeners that were not ultimately employed. In the final implementation, the vessel was supported near the Airy points, not at the ends. Large ports on top are for pumping, and small ports on the top and sides are for alignment access.

was stable against pumpdown to better than $10 \mu \mathrm{m}$. The range of this flexure is only about $\pm 200 \mu \mathrm{m}$, so it must be carefully positioned initially.

The vacuum chamber was fabricated in two parts, one $2.5 \mathrm{~m}$ long, the other $1.5 \mathrm{~m}$ long, and these were bolted together with an O-ring seal. There is a removable top plate for each part, which is O-ring sealed. The end transitions employed O-ring seals to conventional UHV hardware. Most ports were aluminum/stainless steel transition nipples, though O-ring sealed ASA ports were used (on the opposite side in Fig. 9) for the diagnostic pop-ins, to allow some lateral adjustability in the position of the pop-in. The vacuum need only be $10^{-6}$ Torr.

The trim magnets are a square iron core window-frame design, capable of creating $\pm 50 \mathrm{G}$ fields on axis with a full scale current of $\pm 5 \mathrm{~A}$ in the coils. However, because there was inadequate clearance between the trim coils and the BPM ports, we removed the leg of the trim coils on one side of the vacuum vessel. The remaining side opposite is capable of creating adequate trim fields, though these fields are no longer symmetrical transversely. The resulting "C" shaped steering coils were measured with a Hall probe and were shown to maintain the steering necessary, without significant gradients.

Between the support posts and the undulator were fine ball-end adjustment screws that met a cone-slot-plane kinematic mount. There is one cone for all four segments, and the rest of the mounting points are slots or planes. Because of galling, it was found necessary that the mounting points be hardened; we had them ground from nickelbonded tungsten carbide, which is less magnetic than the cobalt-bonded variety. These screws could be used to adjust pitch, roll, and vertical position. The transverse slides or parallelogram flexures control yaw and transverse position. Longitudinal position of the whole device is not controlled, since it is arbitrary. The longitudinal position of each segment is set by a fine screw adjustor between each segment, whose setting is determined when the segments are matched magnetically on the pulsed wire apparatus.

Initially it was hoped that these screws would be used only in the initial in-air alignment of the undulator, so they were accessed by means of miniflange blanked ports in the bottom of the vacuum vessel. However, when it was found that the undulator could move and lose alignment during pumpdown, it was decided to replace the miniflange blanks with rotary feedthroughs, so that post-pumpdown adjustments could be made if necessary.

\section{ALIGNMENT}

Rough alignment was performed by ordinary surveying methods, but precision alignment was done by straightness interferometry using mechanical contacts to the undulator. Direct contact between the interferometer optics, in air, had to be made with the undulator segments. A full description of the alignment process is given in Ref. [13] so we include only a brief summary here. 
Initial alignment, using tooling balls mounted on each segment, brought the axis to straightness within about $\pm 200 \mu \mathrm{m}$. A fixture with slits, referenced to the tooling balls and previously calibrated to the magnetic axis, was used to align the entrance and exit of the undulator to a beam line reference laser, which is also used to calibrate the diagnostic pop-ins. Two laser straightness interferometers, also aligned parallel to the beam line reference laser, are used to achieve less than $\pm 20 \mu \mathrm{m}$ alignment error of the magnetic axes. One interferometer can be moved on a path level with the axis horizontally, and the other is vertically above the axis. A rod with invariant length is used to transfer the distance between the tooling ball and the interferometer, and the interferometer optic may be moved over the entire $4 \mathrm{~m}$ length.

\section{RUN TIME DIAGNOSTICS AND TRAJECTORY CONTROLS}

Initially, we elected to use Ce:YAG (yttrium aluminum garnet) crystals as beam position monitors. When the electron beam strikes a $0.5 \mathrm{~mm}$ thick Ce:YAG crystal, it causes the crystal to fluoresce. We thought that this material would have negligible blooming, but in fact the fluorescence spot bloomed excessively, so we abandoned this approach in favor of an optical transition radiation (OTR) approach, even though the signal intensity is much lower than for Ce:YAG [14].

The diagnostic pop-ins are mounted on the vacuum vessel. The two functions they perform are indicated in Fig. 10. The pop-ins may be set to one of three positions, by air cylinders. In the "out" position, the pop-in probe end is flush with the electron beam pipe, to minimize wakefield effects on the electron beam. In the BPM position, the electron beam creates optical transition radiation on a mirror surface, and this radiation is guided out by means of a periscope to a charge-coupled device (CCD) camera. The resolution of the BPMs is about $20 \mu \mathrm{m}$. In the energy measuring position, light created by the electron beam is guided out to a power detector, in order to generate a gain curve from the eight points where power is monitored.

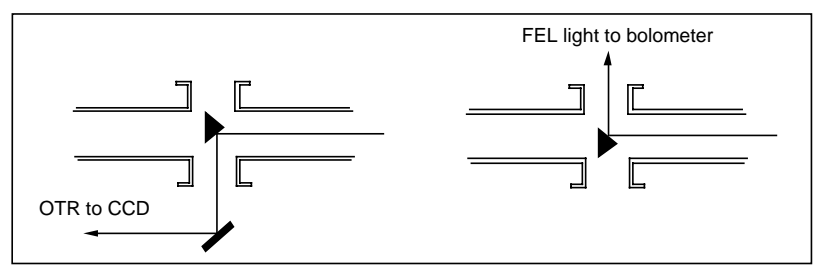

FIG. 10. Schematic layout of diagnostics. On the left, the triangular mirror is positioned in the vacuum chamber generate optical transition radiation (OTR), which is guided out of a periscope to a charge-coupled device (CCD) camera. On the right, the triangular mirror is positioned to guide FEL light to a power monitor.
There are approximately two betatron oscillation periods in the length of the VISA undulator, and the diagnostics and steering trim coils are placed at intervals of roughly $\pi / 2$ phase advance. The trim coils are iron-core pictureframe electromagnets mounted outside the aluminum vacuum vessel. A separate power supply is provided for each pair of coils. Simulations were performed to analyze the effect of run time corrections. We find that errors as large as $200 \mu \mathrm{m}$ can be reduced to $50 \mu \mathrm{m}$, but can require kicks up to $1 \mathrm{mrad}$. The coils are $5 \mathrm{~cm}$ long, axially, so a $1 \mathrm{mrad}$ kick at a beam energy of $85 \mathrm{MeV}$ requires a field of about $57 \mathrm{G}$.

The trim coil power supplies are controlled by the same computer that reads the positions of the beam on the CCD cameras. The pop-ins can be inserted sequentially, the beam positions are acquired, and then a computer algorithm could be used to determine the required changes in steering trim currents. This process could be iterated, as a soft-constraints approach that does not require the beam to pass through the center of each BPM, but only requires the best overall straightness. This beam-based approach is difficult to implement because there are four FODO cells per corrector. Launch positions and angles are monitored by button-type electron BPMs, so that run-time corrections can be made. However, the diagnostics will be used infrequently to align the electron beam in the FEL, so we rely on thermal and mechanical stability in the intervals between recorrection of the steering currents.

\section{SUMMARY}

The VISA FEL is designed to generate $800-600 \mathrm{~nm}$ light from the SASE process. A $4 \mathrm{~m}$ undulator should be adequate to reach saturation at these wavelengths, provided that tolerances on magnetic field errors and alignment can be maintained. The most stringent requirement is that trajectory walk-off be held to less than about $\pm 50 \mu \mathrm{m} /$ gain length. By specifying tight tolerances on the permanent magnets, sorting them, assembling them precisely and shimming, we met this requirement for each segment. By shimming across segment joints, and accurately aligning the segments with straightness interferometers, we met the walk-off requirement for the entire device. Finally, using beam position monitoring and steering trims, remaining trajectory errors can be reduced further. Several problems with the vacuum vessel and supports, and with the diagnostics, were solved in the process of commissioning.

\section{ACKNOWLEDGMENTS}

This work was supported by the United States Department of Energy, Office of Basic Energy Sciences, under Contract No. DE-AC03-76SF00515. The authors are pleased to acknowledge valuable technical contributions from Lorraine Solomon, Jeff Aspenleiter, and Michael Lehecka, of Brookhaven National Laboratory, and Don 
Arnett, Bob Channels, Robert DiMattia, Brian Fuss, Catherine Lecocq, Ben Scott, Mike Swanson, and Ann Trautwein of SLAC.

[1] SLAC Linear Coherent Light Source Design Study Report No. Pub R-521, 1988.

[2] R. Bonifacio, C. Pellegrini, and L. M. Narducci, Opt. Commun. 50, 373 (1984).

[3] S. V. Milton et al., Science 292, 2037 (2001).

[4] A. Tremaine et al., in Proceedings of the 2001 FreeElectron Laser Conference, Darmstadt, Germany (NorthHolland, Amsterdam, 2001).

[5] J. Andruszkow et al., Phys. Rev. Lett. 85, 3825 (2000).

[6] J. Pflueger, Nucl. Instrum. Methods Phys. Res., Sect. A 445, 366 (2000).

[7] M. J. Hogan et al., Phys. Rev. Lett. 81, 4867-4870 (1998).

[8] http://www-ssrl.slac.stanford.edu/visa/VISAHome.html
[9] K. Halbach, Nucl. Instrum. Methods 187, 109 (1981).

[10] S. Lidia and R. Carr, Rev. Sci. Instrum. 66, 1865 (1995).

[11] R. Warren and C. Fortgang, Nucl. Instrum. Methods Phys. Res., Sect. A 341, 444 (1994).

[12] G. Rakowsky, J. Aspenleiter, I. Solomon, R. Carr, R. Ruland, and S. Lidia, in Proceedings of the 1999 Particle Accelerator Conference (PAC99), New York (IEEE, Piscataway, NJ, 1999).

[13] R. Ruland, D. Arnett, G. Bowden, R. Carr, B. Dix, B. Fuss, C. Le Cocq, Z. Wolf, J. Aspenleiter, G. Rakowsky, J. Skaritka, P. Duffy, and M. Libkind, in Proceedings of the 1999 Particle Accelerator Conference (PAC99), New York (Ref. [13]).

[14] I. A. Murokh, P. Frigola, C. Pellegrini, S. Reiche, J. Rosenzweig, A. Tremaine, E. Johnson, X. J. Wong, V. Yakimenko, P. Emma, L. Klaisner, and H.-D. Nuhn, in Proceedings of the 22nd International Free Electron Laser Conference (FEL2000), Durham, NC, 2000 (NorthHolland, Amsterdam, 2000). 